\title{
ESTUDO DA EQUIVALÊNCIA ENTRE AS LISTAS DE SENTENÇAS EM PORTUGUÊS
}

\section{Study of equivalence among the lists of sentences in Portuguese}

\author{
Sinéia Neujahr dos Santos ${ }^{(1)}$, Raquel Cristina Daniel ${ }^{(2)}$, Maristela Julio Costa ${ }^{(3)}$
}

\section{RESUMO}

Objetivo: verificar a equivalência entre as listas de sentenças do teste Listas de Sentenças em Português, proposto por Costa em 1998. Métodos: as informações foram retiradas de um banco de dados de um projeto de pesquisa. Foram selecionados 240 indivíduos que possuíam no mínimo o ensino médio completo, com idade entre 18 e 30 anos e apresentarem limiares de audibilidade dentro da normalidade. Os Limiares de Reconhecimento de Sentenças no Silêncio e no Ruído foram pesquisados em cada orelha separadamente através de fones auriculares. No Limiar de Reconhecimento de Sentenças no Ruído, fala e ruído foram apresentadas ipsilateralmente. Resultados: ao comparar os resultados encontrados entre as listas, obteve-se uma diferença média de 0,03 dB NA a 0,86 dB NA no silêncio e de 0,04 dB NA a 1,06 dB NA no ruído. Observou-se também que os valores mínimos e máximos nas seis listas foram muito próximos. Conclusão: observou-se que na aplicação do teste Listas de Sentenças em Português, através de fones auriculares, tanto no silêncio como no ruído, foram obtidos resultados similares nas listas 1B, 2B, 3B, 4B, 5B e 6B, mostrando haver equivalência entre as listas.

DESCRITORES: Audiologia; Testes de Discriminação da Fala; Percepção Auditiva

\section{INTRODUÇÃO}

A habilidade para compreender a fala pode ser considerada como a mais importante entre os aspectos mensuráveis na avaliação dos distúrbios da comunicação humana ${ }^{1}$. Na avaliação audiológica básica, sílabas e palavras têm sido utilizadas para medir o desempenho auditivo do indivíduo em tarefas de reconhecimento de fala. Entretanto, a utilização de sentenças é considerada o melhor instrumento para avaliar a comunicação do indivíduo no seu dia-a-dia. O desempenho do indivíduo

(1) Fonoaudióloga do Projeto de Promoção da Saúde Auditiva da Universidade Federal de Santa Maria, UFSM, Santa Maria, RS; Mestranda em Distúrbios da Comunicação Humana na Universidade Federal de Santa Maria.

(2) Fonoaudióloga da Clínica Valéria Colomé, RS; Especialista em Fonoaudiologia pela Universidade Federal de Santa Maria; Mestre em Distúrbios da Comunicação Humana pela Universidade Federal de Santa Maria.

(3) Fonoaudióloga; Professora Adjunta do Departamento de Fonoaudiologia da Universidade Federal de Santa Maria, UFSM, Santa Maria, RS; Doutora em Ciências dos Distúrbios da Comunicação Humana pela Universidade Federal de São Paulo.

Conflito de interesses: inexistente frente às frases nos dá uma medida direta de como ele é capaz de participar de uma situação habitual de fala ${ }^{2,3}$.

A apresentação de sentenças, tanto em ambiente silencioso quanto na presença de ruído, representa melhor uma situação de comunicação e assim retratam, de uma forma mais fidedigna, a real condição do indivíduo. Com esses fins, em $1998{ }^{3}$ foi desenvolvido o primeiro teste na língua portuguesa contendo sentenças como estímulo de fala. O teste é constituído de oito Listas de Sentenças em Português Brasileiro, com períodos simples, cuja extensão variou de quatro a sete palavras por sentença. As listas foram denominadas 1A, 1B, 2B, 3B, 4B, 5B, 6B e 7B, foneticamente balanceadas. Também foi desenvolvido um ruído com espectro de fala, que permite determinar os limiares de reconhecimento de sentenças no silêncio e no ruído. As frases representam mais adequadamente características de uma situação conversacional do que palavras isoladas, simulando em ambiente clínico situações similares às do dia-a-dia do indivíduo ${ }^{1}$.

O teste Listas de Sentenças em Português (LSP) possibilita mensurar as habilidades de reconhecimento de fala de um ouvinte, como um reflexo de 
seu desempenho em situações auditivas realistas. Nos últimos anos, diversas pesquisas com objetivos e estratégias diferentes foram realizadas com este material ${ }^{1,2,4-6}$.

Considerando que este teste vem sendo estudado em diferentes populações, mas que a equivalência das listas havia sido investigada somente durante o desenvolvimento do projeto piloto do material, na primeira fase das pesquisas em fita cassete, julga-se necessário avaliar a equivalência entre as listas utilizando a versão do material gravado em $C D$.

Dessa forma o presente estudo tem como objetivo estudar a equivalência entre as listas, a partir dos limiares de reconhecimento de sentenças no silêncio e no ruído comparando as medidas obtidas, na mesma posição de apresentação, variando o grupo de indivíduos normo-ouvintes.

\section{MÉTODOS}

Este estudo foi realizado no Laboratório de Próteses Auditivas do Serviço de Atendimento Fonoaudiológico (SAF) da Universidade Federal de Santa Maria (UFSM).

Os materiais utilizados para efetuar esta pesquisa foram retirados do banco de dados de um projeto de pesquisa intitulado "Valores de referência para o reconhecimento de sentenças no silêncio e no ruído em indivíduos adultos normo-ouvintes", aprovado pelo comitê de ética do Centro de Ciências da Saúde, da Universidade Federal de Santa Maria, cadastro no 016/03, em 2003. Foram selecionados dados de 240 indivíduos que deveriam ter, no mínimo, concluído o ensino médio, ter idade entre 18 e 30 anos e apresentarem limiares de audibilidade dentro da normalidade, ou seja, no máximo 25 dB NA, nas frequências de 250 a $8000 \mathrm{~Hz}^{7}$. Qualquer indivíduo que, por algum motivo, não aceitou participar da pesquisa, não cumpriu os critérios de seleção ou apresentou algum fator que pudesse interferir no teste, como alterações neurológicas e/ ou de fluência verbal, foi excluído da amostra.

Todos os sujeitos que disponibilizaram seus dados para fins de pesquisa assinaram o Termo de Consentimento previamente. Todos os participantes foram submetidos à anamnese, com perguntas referentes aos dados pessoais, nível de escolaridade, história otológica e queixas auditivas e, em seguida, à inspeção visual do meato acústico externo. Após foi realizada audiometria tonal liminar e logoaudiometria.

Para obtenção dos limiares de reconhecimento de sentenças no silêncio (LRSS) e no ruído (LRSR), utilizou-se o material do teste Listas de Sentenças em Português ${ }^{3}$, o qual é constituído por uma lista de 25 sentenças, sete listas de dez sentenças e um ruído de espectro de fala. Este material apresentase gravado em CD, no qual as sentenças e o ruído foram gravados em canais independentes, permitindo sua apresentação tanto no silêncio quanto no ruído. O LRSS e o LRSR foram pesquisados em cada orelha separadamente. Para a obtenção das medidas no ruído, os dois estímulos (fala e ruído) foram apresentados ipsilateralmente.

Os indivíduos foram avaliados em cabine acusticamente tratada, utilizando um audiômetro digital de dois canais, marca Fonix - Hearing Evaluator modelo FA - 12, tipo I e fones auriculares tipo TDH39P, marca Telephonics.

As sentenças foram apresentadas utilizando-se um Compact Disc Player Digital Toshiba - 4149, acoplado ao audiômetro acima descrito.

A saída de cada canal foi calibrada no VU-meter do audiômetro antes de iniciar o teste. $O$ tom de $1000 \mathrm{~Hz}$ presente no mesmo canal do CD em que estão gravadas as sentenças, bem como o ruído mascarante presente no outro canal, foram colocados no nível zero.

Considerando que foi observada pela autora do material, por meio da análise espectrográfica, a existência de uma diferença de $7 \mathrm{~dB}$ entre o volume de gravação dos dois sinais apresentados no CD (fala e ruído), no qual a fala está gravada numa intensidade de $7 \mathrm{~dB}$ abaixo da intensidade do ruído, adotou-se, como procedimento para o cálculo do LRSS e LRSR, a subtração de $7 \mathrm{~dB}$ dos valores de fala registrados e observados no dial do equipamento.

A aplicação do material foi realizada por meio do procedimento denominado "estratégia sequencial, adaptativa ou ascendente-descendente", que permite determinar o limiar de reconhecimento de fala, ou seja, o nível necessário para o indivíduo identificar corretamente em torno de $50 \%$ dos estímulos de fala apresentados em uma determinada condição sinal-ruído ${ }^{8}$.

A estratégia de aplicação do teste consistiu em apresentar o estímulo de fala em uma determinada intensidade, neste caso, na condição de silêncio, a $15 \mathrm{~dB}$ e na condição de ruído, $70 \mathrm{~dB}$. Ao se obter resposta correta, diminuía-se a intensidade de apresentação do estímulo seguinte. Ao se obter resposta incorreta, aumentava-se a intensidade do estímulo seguinte. Os intervalos de apresentação dos estímulos foram de $5 \mathrm{~dB}$ até a primeira mudança no padrão de resposta e, a partir daí, os intervalos de apresentação das sentenças foram de 2,5 dB entre si até o final da lista.

Esse procedimento foi utilizado tanto para medidas de reconhecimento de sentenças no silêncio 
como no ruído. No teste com ruído competitivo, este foi mantido constante em $65 \mathrm{~dB} \mathrm{NA}^{8}$.

A partir da anotação dos níveis de apresentação de cada sentença separadamente, calculou-se a média destes valores para obter o LRSS e o LRSR. Para obter a relação $S / R$, o LRSR foi subtraído da intensidade do ruído competitivo.

É importante salientar que os LRSR foram expressos por meio das relações $S / R$ nas quais os indivíduos foram capazes de reconhecer em torno de $50 \%$ dos estímulos apresentados.

Considerando que o teste foi composto de seis listas formadas por dez sentenças diferentes, escoIneu-se como estratégia a apresentação das listas em ordem diferente, a fim de evitar resultados tendenciosos devido à apresentação de uma determinada lista sempre em uma mesma posição.

Assim sendo, os 240 indivíduos foram divididos em seis grupos, que iniciaram sempre por uma lista diferente, formando, deste modo, diferentes grupos de acordo com a ordem de apresentação das listas de sentenças.

Para $\circ$ Grupo 1, foram apresentadas as listas 1B, 2B, 3B e 4B; para o Grupo 2, as listas 2B, 3B, 4B e 5B; para o Grupo 3, as listas 3B, 4B, 5B e 6B; para o Grupo 4, as listas $4 B, 5 B, 6 B$ e $1 B$; para o Grupo 5, as listas 5B, 6B, 1B e 2B e para o Grupo 6 as listas $6 \mathrm{~B}, 1 \mathrm{~B}, 2 \mathrm{~B}$ e $3 \mathrm{~B}$. É válido mencionar que as duas primeiras listas de cada grupo foram sempre aplicadas no silêncio e as outras duas no ruído.

Para iniciar o teste, todos os indivíduos foram treinados com a lista $1 \mathrm{~A}$, que consiste em uma lista de 25 sentenças, que também fez parte do teste, mas foi utilizada apenas para treinamento. $O$ treinamento foi realizado apresentando sempre, inicialmente no silêncio, as cinco primeiras sentenças da lista $1 \mathrm{~A}$ na orelha esquerda para, então, iniciar a obtenção das medidas propriamente ditas na oreIha direita e depois na orelha esquerda. A primeira lista de cada grupo foi aplicada na orelha direita, a segunda na orelha esquerda, seguida da terceira na orelha direita e a quarta na orelha esquerda.

Após as medidas no silêncio, foram apresentadas, também para treinamento, as sentenças de 5 a 10 da lista $1 \mathrm{~A}$, na presença de ruído na orelha esquerda e, então, apresentada a lista de teste propriamente dita na orelha direita e depois na orelha esquerda.

Estes parâmetros foram seguidos para todos os indivíduos avaliados, variando apenas a ordem de apresentação das listas. As listas de sentenças utilizadas na pesquisa estão na Figura 1.

Esta pesquisa teve aprovação do Comitê de Ética do gabinete de projetos do Centro de Ciências da Saúde sob o número 13894.
Para análise estatística dos dados desta pesquisa, comparando as eventuais diferenças entre as seis listas, utilizou-se a análise de variância (ANOVA) e o teste de comparação Duncan.

O nível de rejeição para a hipótese de nulidade foi fixado em um valor menor ou igual a $5 \%(p<0,05)$. Os resultados estatisticamente significantes foram assinalados com um asterisco $\left({ }^{*}\right)$.

\section{RESULTADOS}

Nas Tabelas 1 e 2 estão expressos as médias aritméticas, os desvios padrão, os valores mínimo e máximo e o resultado do teste de comparação de Duncan, referentes aos LRSS obtidos entre as seis listas, na mesma posição de apresentação, variando o grupo de indivíduos normo-ouvintes, respectivamente da orelha direita e orelha esquerda. Resultados com letras iguais não diferem estatisticamente $(p>0,05)$.

Nas Tabelas 3 e 4 estão expostas as médias aritméticas, os desvios padrão, os valores mínimo e máximo e o resultado do teste de comparação de Duncan, referentes a relação Sinal/Ruído na qual foram obtidos os LRSR entre as seis listas, na mesma posição de apresentação, variando o grupo de indivíduos normo-ouvintes, respectivamente da orelha direita e orelha esquerda. Resultados com letras iguais não diferem estatisticamente $(p>0,05)$.

Foi observado, para a orelha direita, que a lista $6 \mathrm{~B}$ (Tabela 1) possuiu o maior valor médio do LRSS, $6,67 \mathrm{~dB} N \mathrm{NA}$ e a lista $2 \mathrm{~B}$, o menor valor médio 5,85 $\mathrm{dB}$ NA. A diferença numérica entre estas médias foi de $0,82 \mathrm{~dB}$ NA. A mínima diferença entre as listas ocorreu entre a $1 \mathrm{~B}$ e $4 \mathrm{~B}$ que foi de $0,05 \mathrm{~dB} N \mathrm{NA}$, em que $1 \mathrm{~B}$ teve a média de $6,07 \mathrm{~dB}$ NA e 4B de 6,12 dB NA.

A análise de variância encontrou diferenças estatisticamente significantes entre as listas. Estas foram divididas em dois grupos de equivalência: grupo $A$, em que se observaram as seguintes listas $1 \mathrm{~B}, 3 \mathrm{~B}, 4 \mathrm{~B}, 5 \mathrm{~B}$ e $6 \mathrm{~B}$ e grupo $\mathrm{B}$ as listas $3 \mathrm{~B}, 5 \mathrm{~B}, 4 \mathrm{~B}$, $1 B$ e $2 B$, que desempenharam um comportamento estatisticamente da mesma forma.

Apesar de a análise estatística ter subdivido as listas em dois grupos de equivalência, constatouse que existem listas que se repetem nos diferentes grupos, ou seja, nenhuma lista foi considerada totalmente diferente das demais, pois sempre existiu um elo de semelhança entre elas. Por exemplo: as listas $3 \mathrm{~B}, 5 \mathrm{~B}, 4 \mathrm{~B}$ e $1 \mathrm{~B}$ pertenciam aos dois grupos, fazendo o elo com as listas $6 \mathrm{~B}$, que pertencia somente ao grupo $A$, em que havia um elo com a lista $2 \mathrm{~B}$, que pertencia somente ao grupo $\mathrm{B}$. 


\section{LISTA $1 A$}

1. Não posso perder o ônibus.

2. Vamos tomar um cafezinho.

3. Preciso ir ao médico.

4. A porta da frente está aberta.

5. A comida tinha muito sal.

6. Cheguei atrasado para a reunião.

7. Vamos conversar lá na sala.

8. Depois liga pra mim.

9. Esqueci de pagar a conta.

10. Os preços subiram ontem.

11. O jantar está na mesa.

12. As crianças estão brincando.

13. Choveu muito neste fim de semana.

14. Estou morrendo de saudade.

15. Olhe bem ao atravessar a rua.

16. Preciso pensar com calma.

17. Guardei o livro na primeira gaveta.

18. Hoje é meu dia de sorte.

19. O sol está muito quente.

20. Sua mãe acabou de sair de carro.

\section{LISTA 1B}

1. O avião já está atrasado.

2. O preço da roupa não subiu.

3. O jantar da sua mãe estava bom.

4. Esqueci de ir ao banco.

5. Ganhei um carro azul lindo.

6. Ela não está com muita pressa.

7. Avisei seu filho agora.

8. Tem que esperar na fila.

9. Elas foram almoçar mais tarde.

10. Não pude chegar na hora.

\section{LISTA 2B}

1. Acabei de passar um cafezinho.

2. A bolsa está dentro do carro.

3. Hoje não é meu dia de folga.

4. Encontrei seu irmão na rua.

5. Elas viajaram de avião.

6. Seu trabalho estará pronto amanhã.

7. Ainda não está na hora.

8. Parece que agora vai chover.

9. Esqueci de comprar os pães.

10. Ouvi uma música linda.

\section{LISTA 3B}

1. Ela acabou de bater o carro.

2. É perigoso andar nessa rua.

3. Não posso dizer nada.

4. A chuva foi muito forte.

5. Os preços subiram na segunda.

6. Esqueci de levar a bolsa.

7. Os pães estavam quentes.

8. Elas já alugaram uma casa na praia.

9. Meu irmão viajou de manhã.

10. Não encontrei meu filho.

\section{LISTA 4B}

1. Sua mãe pôs o carro na garagem.

2. O aluno quer assistir ao filme.

3. Ainda não pensei no que fazer.

4. Essa estrada é perigosa.

5. Não paguei a conta do bar.

6. Meu filho está ouvindo música.

7. A chuva inundou a rua.

8. Amanhã não posso almoçar.

9. Ela viaja em dezembro.

10. Você teve muita sorte.

\section{LISTA 5B}

1. Depois, a gente conversa.

2. Ela acabou de servir o almoço.

3. Esta carta chegou ontem.

4. Preciso terminar o meu trabalho.

5. Não posso esquecer da mala.

6. A rua estava muito escura.

7. A data do exame foi adiada.

8. Elas alugaram um carro no verão.

9. Minha viagem foi ótima.

10. Eles foram comprar pães.

\section{LISTA 6B}

1. Vou viajar as nove da manhã.

2. Meu irmão bateu o carro ontem.

3. Prometi a ele não contar o segredo.

4. Cheguei atrasada na aula.

5. Esta rua é perigosa.

6. Esqueci da bolsa na sua mesa.

7. Ela comprou os últimos pães.

8. A casa de campo já foi alugada.

9. Os preços não devem subir.

10. Não falei com sua filha.

Figura 1 - Lista de Sentenças em Português (Costa, 1998) 
Tabela 1 - Limiares de Reconhecimento de Sentenças no Silêncio (LRSS), comparando as seis listas de sentenças em português, na orelha direita de cada grupo

\begin{tabular}{ccccccc}
\hline & \multicolumn{3}{c}{ LRSS (dB NA) - Orelha direita } & & \\
\hline Variável & N & Média & Mínimo & Máximo & \multicolumn{2}{c}{ Duncan $^{*}$} \\
\hline Lista 6B Grupo 6 & 40 & 6,67 & 3,55 & 8,55 & $\mathrm{~A}$ & \\
Lista 3B Grupo 3 & 40 & 6,48 & 4,11 & 9,66 & $\mathrm{~A}$ & $\mathrm{~B}$ \\
Lista 5B Grupo 5 & 40 & 6,36 & 3,55 & 9,11 & $\mathrm{~A}$ & $\mathrm{~B}$ \\
Lista 4B Grupo 4 & 40 & 6,12 & 2,44 & 8,55 & $\mathrm{~A}$ & $\mathrm{~B}$ \\
Lista 1B Grupo 1 & 40 & 6,07 & 3,55 & 8,55 & $\mathrm{~A}$ & $\mathrm{~B}$ \\
Lista 2B Grupo 2 & 40 & 5,85 & 3,00 & 8,55 & & $\mathrm{~B}$ \\
\hline
\end{tabular}

* Letras iguais não diferem estatisticamente $(p>0,05)$

Tabela 2 - Limiares de Reconhecimento de Sentenças no Silêncio (LRSS), comparando as seis listas de sentenças em português na orelha esquerda de cada grupo

\begin{tabular}{ccccccc}
\hline & \multicolumn{3}{c}{ LRSS (dB NA) - Orelha esquerda } & & \\
\hline Variável & N & Média & Mínimo & Máximo & \multicolumn{2}{c}{ Duncan $^{*}$} \\
\hline Lista 6B Grupo 1 & 40 & 6,60 & 3,00 & 9,66 & $\mathrm{~A}$ & \\
Lista 4B Grupo 5 & 40 & 6,30 & 3,22 & 8,55 & $\mathrm{~A}$ & $\mathrm{~B}$ \\
Lista 5B Grupo 6 & 40 & 6,14 & 3,55 & 9,66 & $\mathrm{~A}$ & $\mathrm{~B}$ \\
Lista 3B Grupo 4 & 40 & 6,11 & 3,00 & 8,55 & $\mathrm{~A}$ & $\mathrm{~B}$ \\
Lista 1B Grupo 2 & 40 & 5,96 & 3,55 & 8,55 & & $\mathrm{~B}$ \\
Lista 2B Grupo 3 & 40 & 5,74 & 3,00 & 8,00 & & $\mathrm{~B}$ \\
\hline
\end{tabular}

* Letras iguais não diferem estatisticamente $(p>0,05)$

Tabela 3 - Relação Sinal/Ruído, comparando as seis listas de sentenças em português, na orelha direita de cada grupo

\begin{tabular}{cccccccc}
\hline & \multicolumn{9}{c}{ Relação S/R - Orelha direita } & & \\
\hline Variável & N & Média & Mínimo & Máximo & \multicolumn{3}{c}{ Duncan $^{*}$} \\
\hline Lista 5B Grupo 3 & 40 & $-4,79$ & $-3,61$ & $-8,00$ & A & & \\
Lista 6B Grupo 4 & 40 & $-5,14$ & $-3,11$ & $-8,11$ & A & B & \\
Lista 1B Grupo 5 & 40 & $-5,23$ & $-3,11$ & $-8,66$ & A & B & C \\
Lista 2B Grupo 6 & 40 & $-5,31$ & $-3,11$ & $-7,55$ & A & B & C \\
Lista 3B Grupo 1 & 40 & $-5,45$ & $-3,11$ & $-9,22$ & & B & C \\
Lista 4B Grupo 2 & 40 & $-5,85$ & $-4,22$ & $-8,11$ & & & \\
\hline
\end{tabular}

* Letras iguais não diferem estatisticamente $(p>0,05)$

Tabela 4 - Relação Sinal/Ruído, comparando as seis listas de sentenças em português na orelha esquerda de cada grupo

\begin{tabular}{lcccccc}
\hline & \multicolumn{3}{c}{ Relação S/R - Orelha esquerda } & & \multicolumn{2}{c}{ Duncan $^{*}$} \\
\hline Variável & N & Média & Mínimo & Máximo & A & \\
\hline Lista 6B Grupo 3 & 40 & $-4,96$ & $-3,66$ & $-7,00$ & A & \\
Lista 1B Grupo 4 & 40 & $-5,03$ & $-2,55$ & $-7,55$ & A & \\
Lista 3B Grupo 6 & 40 & $-5,27$ & $-3,66$ & $-7,55$ & A & \\
Lista 5B Grupo 2 & 40 & $-5,31$ & $-3,66$ & $-7,44$ & A & B \\
Lista 2B Grupo 5 & 40 & $-5,38$ & $-3,66$ & $-8,66$ & B \\
Lista 4B Grupo1 & 40 & $-5,80$ & $-3,66$ & $-8,66$ & & B \\
\hline
\end{tabular}

* Letras iguais não diferem estatisticamente $(p>0,05)$ 
A análise das seis listas aplicadas na orelha esquerda (Tabela 2) demonstrou a maior diferença numérica entre as médias das listas $6 \mathrm{~B}$ e $2 \mathrm{~B}$ de 0,86 $\mathrm{dB} N \mathrm{~N}$, em que $6 \mathrm{~B}$ teve a média de 6,60 dB NA e 2B de 5,74 dB NA. A menor diferença ocorreu entre as médias das listas $3 B$ e $5 B$ de $0,03 \mathrm{~dB} N A$, em que $3 B$ teve a média de 6,11 dB NA e 5B de 6,14 dB NA.

A análise estatística também encontrou diferença significante entre as seis listas, que foram divididas em dois grupos de equivalência: grupo $A$, com as listas $6 B, 4 B, 5 B$ e $3 B$ e grupo $B$, com a $4 \mathrm{~B}, 5 \mathrm{~B}, 3 \mathrm{~B}, 1 \mathrm{~B}$ e 2B. Nesta condição, as listas que pertenciam aos dois grupos foram a $4 B, 5 B$ e $3 B$, formaram a ligação entre as listas $6 \mathrm{~B}$ do grupo $\mathrm{A}$ e 1B e 2B do grupo B.

Ao observar novamente os resultados da orelha direita, a análise numérica (Tabela 3 ) demonstrou a maior diferença entre as médias nas listas $5 \mathrm{~B}$ e 4B, que foi de 1,06 dB NA, em que 5B teve a média de $-4,79 \mathrm{~dB}$ NA e a 4B de $-5,85 \mathrm{~dB}$ NA. A mínima diferença entre as listas ocorreu entre a $1 \mathrm{~B}$ e $2 \mathrm{~B}$ que foi de $0,08 \mathrm{~dB}$ NA, em que $1 \mathrm{~B}$ teve a média de $-5,23$ dB NA e 2B de $-5,31$ dB NA.

A análise de variância encontrou diferenças estatisticamente significantes entre as seis listas e, então, estas foram divididas em três grupos de equivalência: grupo $A$, com as listas $5 B, 6 B, 1 B$ e $2 \mathrm{~B}$, grupo $\mathrm{B}$, com as listas $6 \mathrm{~B}, 1 \mathrm{~B}, 2 \mathrm{~B}$ e $3 \mathrm{~B}$ e grupo $C$, com as listas $2 B, 3 B$ e $4 B$. Observou-se que a lista $2 \mathrm{~B}$ esteve presente nos três grupos, fazendo a ligação entre as demais listas.

A análise numérica das médias obtidas na oreIha esquerda (Tabela 4) demonstrou a maior diferença entre as médias nas listas $6 \mathrm{~B}$ e $4 \mathrm{~B}$ de 0,84 $\mathrm{dB} N A$, em que $6 \mathrm{~B}$ teve a média de $-4,96 \mathrm{~dB} N \mathrm{NA}$ e $4 B$ de $-5,80 \mathrm{~dB} N \mathrm{NA}$. A menor diferença ocorreu entre as médias das listas 5B e 3B de 0,04 dB NA, em que $5 B$ teve a média de $-5,31 \mathrm{~dB}$ NA e $3 B$ de $-5,27$ dB NA.

A análise estatística também encontrou diferença significante entre as seis listas, que foram divididas em dois grupos: grupo $\mathrm{A}$, com as listas $6 \mathrm{~B}$, 1B, 3B e 5B e 2B e grupo B, com 4B e 2B. Constatou-se que a lista $2 \mathrm{~B}$ também se encontra como elo entre as demais listas.

\section{DISCUSSÃO}

Foi verificado que as diferenças observadas entre as seis LSP, tanto no silêncio quanto no ruído, foram pequenas. A maior diferença média entre as listas foi de 1,06 dB, no ruído, sendo que as demais estão abaixo de $1 \mathrm{~dB}$. Também os valores mínimos e máximos nas seis listas foram muito próximos.

Alguns estudos foram realizados com o mesmo objetivo de verificar a equivalência das listas e a confiabilidade dos testes de fala no silêncio e no ruído.

Em 1979, pesquisadore ${ }^{9}$ analisaram a equivalência e confiabilidade do teste de fala com frases Hearing in Noise Test (HINT), desenvolvidas por eles. Eles aplicaram 10 listas com 13 sentenças cada em adultos com limiares dentro dos padrões de normalidade e as medidas obtidas no testereteste com diferentes listas mostraram resultados que diferiram em aproximadamente $1 \mathrm{~dB}$.

Em um trabalho realizado em $1994{ }^{10}$, foi desenvolvido outro teste, o Hearing in Noise Test (HINT), composto por um conjunto de 21 listas de frases com 11 sentenças cada e um ruído para a predição do limiar de sentenças no silêncio e no ruído, também se testou a equivalência e confiabilidade de seu teste. As palavras foram foneticamente balanceadas e o ruído foi desenvolvido com o mesmo espectro de frequência das frases. A confiabilidade do teste e a equivalência entre as listas também foi comprovada, variando em torno de $1 \mathrm{~dB}$.

Já em 2000, outra pesquisa ${ }^{11}$ testou a equivalência de seu teste de fala com sentenças, o Hearing in Noise Test (HINT), e a confiabilidade no teste-reteste de 10 listas com 10 frases apresentadas concomitante com ruído babble em várias relações sinal-ruído, sendo que cinco frases foram apresentadas em cada orelha. 20 indivíduos com audição normal e $20 \mathrm{com}$ perda auditiva neurossensorial foram testados. Após análise foi constatado que as listas 1, 2 e 9; 3, 4 e 5 são equivalentes e que variaram menos que $1 \mathrm{~dB}$ entre elas.

Foi criado em $2005^{12}$ um teste de fala no ruído em uma versão em cantonês, o Cantonese Hearing in Noise Test, baseada na metodologia de outro teste ${ }^{10}$. Foram produzidas 24 listas com dez sentenças cada. 142 indivíduos com audição normal participaram do estudo, sendo que a avaliação foi realizada monoauralmente. Houve baixa variabilidade de respostas dentro de cada lista e constatada alta-confiabilidade inter listas, mostrando que o limiar pode ser obtido confiavelmente utilizando qualquer lista ou conjunto de sentenças.

Em $2008{ }^{13}$, o teste de fala no ruído em espanhol latino americano, o Latin American Spanish Hearing in Noise Test publicou um estudo, no qual participaram México, Colômbia e Argentina. Após as sentenças serem criadas e analisadas, elas foram divididas em três grupos e aplicadas em dez sujeitos normo-ouvintes de cada país, cinco rodadas em cada participante. Após as sentenças muito fáceis ou muito difíceis terem sido excluídas, restaram 287 sentenças, que foram divididas em 24 listas, todas equivalentes entre si, com uma variação de $2,5 \mathrm{~dB}$ entre elas. 
No Brasil, outro teste de fala no ruído, The Brazilian Portuguese Hearing in Noise Test, foi desenvolvido e seus estudos publicados em $2008{ }^{14}$. Posteriormente a criação de 24 listas com dez sentenças cada, 29 sujeitos adultos foram avaliados no silêncio e no ruído. O teste-reteste demonstrou valores de variação de $2,9 \mathrm{~dB}$ no silêncio e 2,3 no ruído.

Em outro trabalho realizado em $2008{ }^{15}$, pesquisadores aplicaram o teste de fala no ruído em turco, o Turkish Hearing in Noise Test, em 30 participantes normo-ouvintes. Após concluírem o processo de criação e análise da confiabilidade, 10 listas foram avaliadas e a situação de retestagem demonstrou resultados de 3,1 dB de variação no silêncio e 2,0 dB no ruído.

Em $2008{ }^{16}$, na Bulgária, uma pesquisa foi executada com o teste de fala no ruído, o Bulgarian Hearing in Noise Test, constituído de 24 listas com dez sentenças. Em seguida a determinação da variabilidade, 18 indivíduos foram avaliados e reavaliados no silêncio e no ruído, variando as listas. Foram encontrados resultados variáveis no silêncio e no ruído, de 2,1 dB e 1,2 dB respectivamente.

$\mathrm{Na}$ França em $2008{ }^{17}$, um estudo realizado com o teste de inteligibilidade de fala no ruído, o French Sentence Test for Speech Intelligibility in Noise (FIST) determinou uma variação inter listas de aproximadamente $1 \mathrm{~dB}$. 20 pessoas foram retestadas utilizando uma combinação das 14 listas. Os valores ficaram próximos de $1 \mathrm{~dB}$.

Foram publicados resultados em $2008{ }^{18}$ sobre teste de fala no ruído em norueguês, o Norwegian
Hearing in Noise Test, no qual 24 listas contendo dez sentenças foram aplicadas e reaplicadas em 60 sujeitos. Os resultados variaram em torno de $1 \mathrm{~dB}$.

Em $2005^{5}$, foi realizado um estudo sobre a confiabilidade do teste LSP e os resultados demonstraram-se altamente confiáveis com relação positiva forte, quando comparados os resultados obtidos em diferentes sessões de avaliação em um grupo de indivíduos jovens normo-ouvintes.

Como se pode observar, resultados muitos semelhantes aos encontrados no presente estudo foram confirmados ou ficaram muito próximos aos encontrados por outros pesquisadores, realizadas em vários países.

Com base nos resultados encontrados em $2005{ }^{5}$ e no presente estudo, o teste Listas de Sentenças em Português ${ }^{3}$ proporciona resultados confiáveis, independente de qual lista de sentenças for utilizada no momento da avaliação.

\section{CONCLUSÃO}

Portanto, a partir deste estudo, observou-se que na aplicação do teste LSP através de fones auriculares, tanto no silêncio como no ruído, foram obtidos resultados similares nas listas $1 \mathrm{~B}, 2 \mathrm{~B}, 3 \mathrm{~B}, 4 \mathrm{~B}$, $5 B$ e $6 B$, mostrando haver equivalência entre as mesmas.

Pode-se, portanto, verificar que as seis listas aqui analisadas, podem ser usadas para avaliação do reconhecimento de fala, tanto na condição de silêncio como no ruído.

\begin{abstract}
Purpose: to investigate the equivalence among of sentence lists of the test: Lists of Sentences, in Portuguese, proposed by Costa in 1998. Methods: the information was taken from a database of a research project. 240 individuals with at least complete high-school, 18 to 30 -year old and having normal audibility thresholds, were studied. The Sentence Recognition Thresholds under Silence and under Noise were separately searched in each ear through earphones. In The Sentence Recognition Thresholds under the Noise, speech and noise were presented at the same ear. Results: comparing the results among the lists, we find average difference of $0.03 \mathrm{~dB} N A$ to $0.86 \mathrm{~dB}$ NA under silence and $0.04 \mathrm{~dB}$ NA to $1.06 \mathrm{~dB}$ NA under noise. The minimum and maximum values in the six lists were very close. Conclusion: it was observed that in the application of the test Lists of Sentences in Portuguese, through earphones, both under silence as well as under noise, similar results were obtained in the lists $1 \mathrm{~B}, 2 \mathrm{~B}, 3 \mathrm{~B}, 4 \mathrm{~B}, 5 \mathrm{~B}$ and $6 \mathrm{~B}$, showing equivalence among the lists.
\end{abstract}

KEYWORDS: Audiology; Speech Discrimination Tests; Auditory Perception 


\section{REFERÊNCIAS}

1. Miranda EC, Costa MJ. Reconhecimento de sentenças no silêncio e no ruído de indivíduos jovens adultos normo-ouvintes em campo livre. Fono Atual. 2006; 8(35):4-12.

2. Soncini F, Costa MJ, Oliveira TMT, Lopes LFD. Correlação entre limiares de reconhecimento de sentenças no silêncio e limiares tonais. Rev Bras Otorrinolaringol. 2003; 69(5):672-7.

3. Costa MJ. Lista de sentenças em português: apresentação e estratégias de aplicação na audiologia. Santa Maria: Pallotti; 1998. p. 26-36.

4. Daniel RC. Limiares de reconhecimento de sentenças no silêncio e no ruído em adultos jovens normo-ouvintes: valores de referência [dissertação]. Santa Maria (RS): Universidade Federal de Santa Maria; 2004.

5. Freitas CD, Lopes LFD, Costa MJ. Confiabilidade dos limiares de reconhecimento de sentenças no silêncio e no ruído. Rev Bras Otorrinolaringol. 2005; 71(5):624-30.

6. Henriques MO, Costa MJ. Limiares de reconhecimento de sentenças, em campo livre, na presença de ruído incidente de diferentes ângulos. Fono Atual. 2006; 38(7):12-24.

7. Davis $\mathrm{H}$, Silverman SR. Interpretação dos resultados da avaliação audiológica. In: Santos TMM, Russo ICP. Prática da audiologia clínica. 6. ed. São Paulo: Cortez; 2007. p.291-310.

8. Levitt $H$, Rabiner LR. Use of a sequential strategy in intelligibility testing. J Acoust Soc Am. 1967; 42(3):609-12.
9. Plomp R, Mimpen AM. Improving the reliability of testing the speech reception threshold for sentences. Audiology. 1979; 18(1):43-52.

10. Nilsson M, Soli SD, Sullivan JA. Development of the Hearing in Noise Test for the measurement of speech reception thresholds in quiet and in noise. $J$ Acoust Soc Am. 1994; 95(2):1085-99.

11. Wong LLN, Soli SD. Development of the Cantonese Hearing In Noise Test (CHINT). Ear Hear. 2005; 26(3):276-89.

12. Bentler RA. List equivalency and test-retest reliability of the Speech in Noise test. Am J Audiol. 2000; 9(2):84-100.

13. Otero CB, Brik G, Flores L, Ortiz S, Abdala C. The Latin American Spanish Hearing in Noise Test. Int J Audiol. 2008; 47(6):362-3.

14. Bevilacqua MC, Banhara MR, Costa EA, Vignoly $A B$, Alvarenga KF. The Brazilian Portuguese hearing in noise test. Int J Audiol. 2008; 47(6):364-5.

15. Cekic S, Sennaroglu G. The Turkish hearing in noise test. Int J Audiol. 2008; 47(6):366-8.

16. Lolov SR, Raynov AM, Boteva IB, Edrev GE. The Bulgarian hearing in noise test. Int $\mathrm{J}$ Audiol. 2008; 47(6):371-2.

17. Luts H, Boon E, Wable J, Wouters J. FIST: a French sentence test for speech intelligibility in noise. Int J Audiol. 2008; 47(6):373-4.

18. Myhrum M, Moen I. The Norwegian hearing in noise test. Int J Audiol. 2008; 47(6):377-8.

RECEBIDO EM: 20/06/2008

ACEITO EM: 09/07/2009

Endereço para correspondência:

Sinéia Neujahr dos Santos

Rua Dom Ático da Rocha, 245

Santa Maria - RS

CEP: 97060-090

E-mail: sineians@gmail.com 